\title{
Dynamically assisted Sauter-Schwinger effect in inhomogeneous electric fields
}

\author{
Christian Schneider and Ralf Schützhold \\ Fakultät für Physik, Universität Duisburg-Essen, \\ Lotharstrasse 1, 47057 Duisburg, Germany \\ E-mail: christian.schneider@uni-due.de, ralf.schuetzhold@uni-due.de
}

ABSTRACT: Via the world-line instanton method, we study electron-positron pair creation by a strong (but sub-critical) electric field of the profile $E / \cosh ^{2}(k x)$ superimposed by a weaker pulse $E^{\prime} / \cosh ^{2}(\omega t)$. If the temporal Keldysh parameter $\gamma_{\omega}=m \omega /(q E)$ exceeds a threshold value $\gamma_{\omega}^{\text {crit }}$ which depends on the spatial Keldysh parameter $\gamma_{k}=m k /(q E)$, we find a drastic enhancement of the pair creation probability - reporting on what we believe to be the first analytic non-perturbative result for the interplay between temporal and spatial field dependences $E(t, x)$ in the Sauter-Schwinger effect. Finally, we speculate whether an analogous effect (drastic enhancement of tunneling probability) could occur in other scenarios such as stimulated nuclear decay, for example.

KEYwords: Nonperturbative Effects, Solitons Monopoles and Instantons

ARXIV EPRINT: 1407.3584 


\section{Contents}

1 Introduction 1

2 World-line instanton method 2

$\begin{array}{llr}3 & \text { Sum of Sauter pulses } & 2\end{array}$

4 Tunneling probability 4

$\begin{array}{lll}5 & \text { Numerical evaluation } & 6\end{array}$

$\begin{array}{llr}6 & \text { Conclusions } & 7\end{array}$

$\begin{array}{llr}7 & \text { Outlook } & 8\end{array}$

\section{Introduction}

Despite the tremendous progress of quantum field theory as a fundamental description of nature, our understanding of its non-perturbative properties is still disappointingly incomplete. In quantum electrodynamics (QED), for example, a striking non-perturbative phenomenon is the Sauter-Schwinger effect predicting the creation of electron-positron pairs out of the vacuum by a strong electric field [1-5]. In case of a constant (sub-critical) electric field $E<E_{\text {crit }}$, the pair creation probability behaves as $(\hbar=c=1)$

$$
P_{e^{+} e^{-}} \sim \exp \left\{-\pi \frac{m^{2}}{q E}\right\}=\exp \left\{-\pi \frac{E_{\text {crit }}}{E}\right\}
$$

where $E_{\text {crit }}=m^{2} / q \approx 1.3 \times 10^{18} \mathrm{~V} / \mathrm{m}$ denotes the Schwinger critical field. Unfortunately, the dependence of this non-perturbative phenomenon on the field profile $\boldsymbol{E}(t, \boldsymbol{r})$ away from the constant field approximation is still mostly terra incognita. There are many results for fields which depend on one coordinate only, such as space $x$ or time $t$ (see, e.g., [6-20]), one of the light-cone coordinates $x_{ \pm}=t \pm x$ (see, e.g., [21-28]), or other linear combinations of $x$ and $t$ [29]. In these cases, the underlying (Dirac or Klein-Fock-Gordon) equation simplifies to an ordinary differential equation (allowing for a WKB approach, for example, see also [30-34]).

However, to the best of our knowledge, there are no analytic non-perturbative results for fields $E(t, x)$ which genuinely depend on space $x$ and time $t$. So far, this case has only been treated numerically via the Wigner formalism (see, e.g., [35-37]) or a direct integration of the Dirac equation (see, e.g., [38-40]). This lack of understanding is not only unsatisfactory from a theoretical point of view. A deeper insight into the impact of space-time dependent fields is also highly desirable in view of experimental efforts with 
lasers [41-51], for example, aiming at a verification of this non-perturbative pair-creation effect. ${ }^{1}$ In the following, we venture a first step into this direction and employ the worldline instanton technique (see, e.g., [52-60]) in order to study the superposition of a spatial and a temporal field pulse as an example for a genuinely space-time dependent field.

\section{World-line instanton method}

Let us start with a brief review of the world-line instanton method, see, e.g., [52-60]. Since the electron spin does not affect the exponent of the pair creation probability [55], we consider the vacuum persistence amplitude of scalar QED

$$
\left\langle 0_{\text {out }} \mid 0_{\text {in }}\right\rangle=\iint \mathcal{D} \phi \mathcal{D} \phi^{*} e^{i \int \mathrm{d}^{4} x\left(\left|D_{\mu} \phi\right|^{2}-m^{2}|\phi|^{2}\right)},
$$

with the covariant derivative $D_{\mu}=\partial_{\mu}+i q A_{\mu}$. After analytic continuation to Euclidean space, this functional path integral can be translated into the world-line representation [52] where $\mathcal{D} \phi \mathcal{D} \phi^{*}$ is replaced by the sum over all closed loops $x_{\mu}(s)$ in Euclidean space. Then, via the saddle point method (with the electron mass $m$ playing the role of the large expansion parameter), the pair creation probability can be estimated as

$$
P_{e^{+} e^{-}}=1-\left|\left\langle 0_{\text {out }} \mid 0_{\text {in }}\right\rangle\right|^{2} \sim e^{-\mathcal{S}},
$$

with the world-line instanton action ${ }^{2}$

$$
\mathcal{S}=m a+i q \int_{0}^{1} \mathrm{~d} s \dot{x}^{\mu} A_{\mu}\left(x^{\nu}\right) .
$$

Here $\dot{x}_{\mu}=\mathrm{d} x_{\mu} / \mathrm{d} s$ denotes the proper-time derivative of a closed $x_{\mu}(s=0)=x_{\mu}(s=1)$ world-line loop $x_{\mu}(s)$ as a solution of the instanton equations

$$
m \ddot{x}_{\mu}=i q F_{\mu \nu} \dot{x}^{\nu} a
$$

with $\ddot{x}_{\mu}=\mathrm{d}^{2} x_{\mu} / \mathrm{d} s^{2}$ and $\dot{x}_{\nu} \dot{x}^{\nu}=a^{2}=$ const.

\section{Sum of Sauter pulses}

Now let us apply the world-line instanton method to a space-time dependent electric field

$$
\boldsymbol{E}(t, x)=\left(\frac{E}{\cosh ^{2}(k x)}+\frac{E^{\prime}}{\cosh ^{2}(\omega t)}\right) \boldsymbol{e}_{x}
$$

consisting of a strong spatial Sauter [1] pulse $\propto E$ and a weaker temporal Sauter pulse $\propto E^{\prime}$ where both field strengths are sub-critical $E^{\prime} \ll E \ll E_{\text {crit }}=m^{2} / q$. Furthermore,

\footnotetext{
${ }^{1}$ Apart from the laser laboratories BELLA (Berkley, USA) and VULCAN (Oxford, UK), which are approaching the strong-field (non-linear) QED regime, we would like to mention the European ELI program, the Russian XCELS initiative, or the Chinese SIOM facility, for example.

${ }^{2}$ Although one could also consider complex instantons, $x^{\mu}(s)$ is purely real in our case while $\hat{A}_{\mu}$ is purely imaginary, cf. eq. (3.3).
} 
in order to be in the non-perturbative regime, we assume slowly varying pulses $\omega, k \ll m$. For convenience, we introduce the spatial and temporal Keldysh [61] parameters via

$$
\gamma_{k}=\frac{m k}{q E}, \gamma_{\omega}=\frac{m \omega}{q E} .
$$

It will be most convenient to represent the spatial pulse by the scalar potential $A_{0}(x)$ but the temporal pulse by the vector potential $A_{1}(t)$. Then, after Wick rotation, the Euclidean vector potential reads

$$
A_{0}\left(x_{1}\right)=i \frac{E}{k} \tanh \left(k x_{1}\right), A_{1}\left(x_{0}\right)=i \frac{E^{\prime}}{\omega} \tan \left(\omega x_{0}\right),
$$

with $x_{0}=i t$ and $x_{1}=x$ as well as $A_{2}=A_{3}=0$. As a result, the instanton equations (2.4) assume the form

$$
\begin{aligned}
& \ddot{x}_{0}=+\frac{q E a}{m}\left(\frac{1}{\cosh ^{2}\left(k x_{1}\right)}-\frac{E^{\prime}}{E} \frac{1}{\cos ^{2}\left(\omega x_{0}\right)}\right) \dot{x}_{1}, \\
& \ddot{x}_{1}=-\frac{q E a}{m}\left(\frac{1}{\cosh ^{2}\left(k x_{1}\right)}-\frac{E^{\prime}}{E} \frac{1}{\cos ^{2}\left(\omega x_{0}\right)}\right) \dot{x}_{0},
\end{aligned}
$$

and are analogous to the planar motion of a charged particle in a magnetic field $\boldsymbol{B}(\boldsymbol{r})=$ $B(x, y) \boldsymbol{e}_{z}$.

Due to $E^{\prime} / E \ll 1$, the second term is negligible unless $\cos ^{2}\left(\omega x_{0}\right)$ becomes very small - which happens near the poles of $E\left(x_{0}, x_{1}\right)$ at $\omega x_{0}= \pm \pi / 2$. Away from these poles, we may omit the second term and the above equations can be integrated approximately to

$$
\begin{aligned}
& \dot{x}_{0}=\frac{a}{\gamma_{k}} \tanh \left(k x_{1}\right)+a b, \\
& \dot{x}_{1}= \pm a \sqrt{1-\left(\frac{\tanh \left(k x_{1}\right)}{\gamma_{k}}+b\right)^{2}} .
\end{aligned}
$$

As mentioned after eq. (2.4), the constant $a$ is given by $\dot{x}_{\nu} \dot{x}^{\nu}=a^{2}=$ const. The other integration constant $b$ determines the velocity $\dot{x}_{0}$ just before (or just after) crossing the $x_{0}$-axis, see figure 1 .

Near the poles $\omega x_{0} \approx \pm \pi / 2$, on the other hand, the second term becomes important. Similar to the reflection of a charged particle at the region of a very strong magnetic field, the instanton trajectory is basically reflected by the "wall" at $\omega x_{0} \approx \pm \pi / 2$ if it reaches out far enough. Since this reflection occurs during a very short proper time $\Delta s$, we may neglect the regular terms in eq. (3.4) and keep only the divergent contributions. Then, the equation for $x_{1}$ can be integrated approximately to

$$
\dot{x}_{1} \approx \frac{q E^{\prime} a}{m \omega} \tan \left(\omega x_{0}\right)+\dot{x}_{1}^{\text {in }},
$$

and thus the equation for $x_{0}$ becomes

$$
\ddot{x}_{0} \approx-\frac{\left(q E^{\prime} a\right)^{2}}{m^{2} \omega} \frac{\tan \left(\omega x_{0}\right)}{\cos ^{2}\left(\omega x_{0}\right)} \sim \frac{1}{\left(\omega x_{0} \pm \pi / 2\right)^{3}} .
$$

As a result, the perpendicular velocity $\dot{x}_{0}$ is reversed by that reflection while the parallel velocity $\dot{x}_{1}$ has the same value $\dot{x}_{1}^{\text {in }}$ before and after the reflection, see figure 1 . 


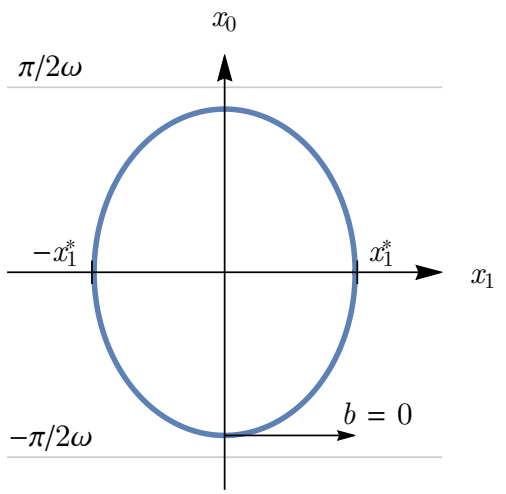

(a) $\omega<\omega^{\text {crit }}$

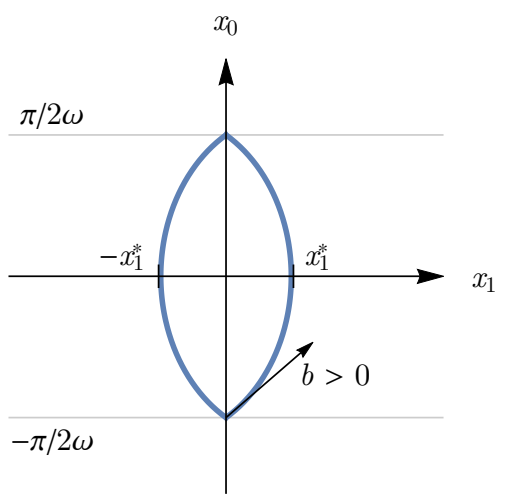

(b) $\omega>\omega^{\text {crit }}$

Figure 1. Sketch of the instanton trajectories in the Euclidean $x_{0}, x_{1}$-plane: Left (a): for frequencies below threshold $\omega<\omega^{\text {crit }} \leftrightarrow \gamma_{\omega}<\gamma_{\omega}^{\text {crit }}$ with $\gamma_{\omega}^{\text {crit }}$ being given by eq. (4.6), the trajectory does not reach the poles at $\pm \pi /(2 \omega)$ and thus it has nearly the same form as in the absence of the temporal pulse $\propto E^{\prime}$. Right (b): for frequencies above threshold $\omega>\omega^{\text {crit }} \leftrightarrow \gamma_{\omega}>\gamma_{\omega}^{\text {crit }}$, the instanton trajectory is effectively reflected at the poles.

\section{Tunneling probability}

From eq. (3.7), we may estimate the contribution to the instanton action (2.3) stemming from the short proper-time period of the reflection and obtain that it is suppressed as $\left(E^{\prime} / E\right) \ln \left(E^{\prime} / E\right)$ for small $E^{\prime}$. Thus, for $E \gg E^{\prime}$, we may approximate the instanton action by the part between the reflections

$$
\mathcal{S} \approx m a-\frac{q E}{k} \int_{0}^{1} \mathrm{~d} s \tanh \left(k x_{1}\right) \dot{x}_{0} .
$$

In order to calculate the above integral, we split the closed loop in figure 1(b) into four quarters: starting at the lower reflection point $x_{0}=-\pi /(2 \omega)$ with $x_{1}=0$, we go to the spatial turning point $x_{1}^{*}$ (where $x_{0}=0$ ), then from there to the upper reflection point $x_{0}=+\pi /(2 \omega)$ with $x_{1}=0$, and then to $-x_{1}^{*}$ (where $x_{0}=0$ ), and finally back to $x_{1}=0$ and $x_{0}=-\pi /(2 \omega)$. Since each quarter yields the same contribution, we get

$$
\mathcal{S} \approx m a-\frac{4 m}{\gamma_{k}} \int_{0}^{x_{1}^{*}} \mathrm{~d} x_{1} \frac{\tanh \left(k x_{1}\right)\left(\tanh \left(k x_{1}\right)+\gamma_{k} b\right)}{\sqrt{\gamma_{k}^{2}-\left(\tanh \left(k x_{1}\right)+\gamma_{k} b\right)^{2}}},
$$

where $x_{1}^{*}$ denotes the spatial turning point given by

$$
\tanh \left(k x_{1}^{*}\right)+\gamma_{k} b=\gamma_{k}
$$


i.e., the zero of the square root in the integral in eq. (4.2) where $\mathrm{d} x_{1} / \mathrm{d} x_{0}=0$. The constant $a$ is determined by $\dot{x}_{\nu} \dot{x}^{\nu}=a^{2}$ and $x_{\mu}(s=0)=x_{\mu}(s=1)$ which gives

$$
a=\frac{4}{\gamma_{k}} \int_{0}^{x_{1}^{*}} \frac{\mathrm{d} x_{1}}{\sqrt{\gamma_{k}^{2}-\left(\tanh \left(k x_{1}\right)+\gamma_{k} b\right)^{2}}} .
$$

The remaining integration constant $b$ depends on the frequency $\omega$. If $\omega$ is too small and thus the poles at $\omega x_{0}= \pm \pi / 2$ are too far away, the instanton trajectory is not reflected at all and thus we have $b=0$, see figure 1 . In case of reflection, the integration constant $b$ is non-zero and determined by the implicit condition

$$
\frac{4 m}{\gamma_{k}} \int_{0}^{x_{1}^{*}} \mathrm{~d} x_{1} \frac{\tanh \left(k x_{1}\right)+\gamma_{k} b}{\sqrt{\gamma_{k}^{2}-\left(\tanh \left(k x_{1}\right)+\gamma_{k} b\right)^{2}}}=\frac{\pi}{2 \omega} .
$$

Together with the above equations for $x_{1}^{*}, a$, and $b$, eq. (4.2) is the main result of this paper.

The threshold condition $b=0$ translates into

$$
\gamma_{\omega}=\frac{\pi}{2} \frac{\gamma_{k} \sqrt{1-\gamma_{k}^{2}}}{\arcsin \left(\gamma_{k}\right)} \stackrel{\text { Def }}{=} \gamma_{\omega}^{\text {crit }} .
$$

Again, if the frequency is too low $\gamma_{\omega}<\gamma_{\omega}^{\text {crit }}$, the instanton trajectory is basically not affected by the poles at $\omega x_{0}= \pm \pi / 2$ leading to $b=0$ and thus the weak temporal pulse $\propto E^{\prime}$ has negligible impact. In this case $b=0$, we get $x_{1}^{*}=\operatorname{artanh}\left(\gamma_{k}\right) / k$ and all the integrals can be carried our analytically, yielding the same results as for a static Sauter pulse, see, e.g., [55]. If the frequency exceeds this threshold value $\gamma_{\omega}>\gamma_{\omega}^{\text {crit }}$, on the other hand, the instanton trajectory is reflected at the poles (i.e., $b>0$ ) and thus the instanton action (4.2) is reduced by the weak temporal pulse $\propto E^{\prime}$, leading to a significant enhancement of the pair creation probability. This enhancement is an example of the dynamically assisted Sauter-Schwinger effect, see [67-72], for an inhomogeneous electric field. In the homogeneous limit $\gamma_{k} \downarrow 0$, the threshold value (4.6) approaches $\gamma_{\omega}^{\text {crit }}=\pi / 2$ consistent with the results of [67]. For $\gamma_{k} \uparrow 1$, the threshold $\gamma_{\omega}^{\text {crit }}$ scales as $\gamma_{\omega}^{\text {crit }} \approx \sqrt{1-\gamma_{k}^{2}}$, i.e., very small frequencies $\omega$ can have a significant impact in this case.

Unfortunately, due to the implicit nature of the condition for $b$, we cannot provide a closed analytical expression for $\mathcal{S}$. However, near but above threshold, we can expand the involved quantities and obtain the following approximate formula for the instanton action:

$$
\mathcal{S}=\frac{m^{2}}{q E}\left\{\begin{array}{rr}
\frac{2 \pi}{1+\sqrt{1-\gamma_{k}^{2}},} & \text { for } \gamma_{\omega} \leq \gamma_{\omega}^{\text {crit }}, \\
\frac{2 \pi}{1+\sqrt{1-\gamma_{k}^{2}}-\pi \frac{\left(1-\gamma_{k}^{2}\right)^{3 / 2}}{\gamma_{k}^{2}\left(\gamma_{\omega}^{\text {crit }}\right)^{4}}\left[\gamma_{\omega}-\gamma_{\omega}^{\text {crit }}\right]^{2}} & \\
+\mathcal{O}\left(\left[\gamma_{\omega}-\gamma_{\omega}^{\text {crit }}\right]^{3}\right), & \text { for } \gamma_{\omega}>\gamma_{\omega}^{\text {crit }} .
\end{array}\right.
$$

The first line (valid below and at threshold $\gamma_{\omega} \leq \gamma_{\omega}^{\text {crit }}$ ) is just the result in the static case (see, e.g., [55]). For $\gamma_{k}=0$, we recover Schwinger's result [5] for a constant field (1.1). Above threshold $\gamma_{\omega}>\gamma_{\omega}^{\text {crit }}$ on the other hand, the action is reduced by the second-order term $\propto\left[\gamma_{\omega}-\gamma_{\omega}^{\text {crit }}\right]^{2}$. 

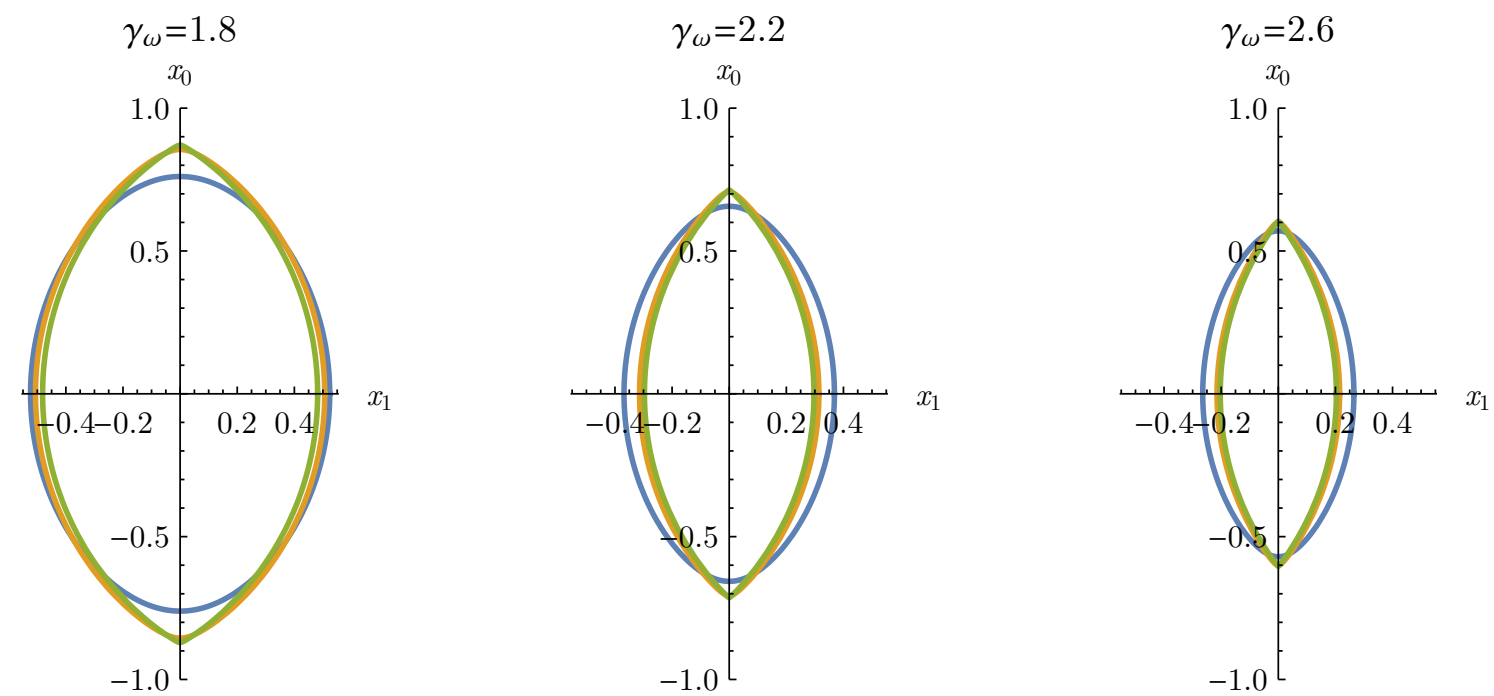

Figure 2. Instanton trajectories [in units of $m /(q E)$ ] found by numerically integrating (3.4) for different values of $\gamma_{\omega}$ and $\gamma_{k}=0.5$ in all cases. The colors represent $E^{\prime} / E=10^{-1}$ (blue), $E^{\prime} / E=10^{-2}$ (orange), and $E^{\prime} / E=10^{-3}$ (green). The limit $E^{\prime} / E \rightarrow 0$ used in the analytic approximation (cf. figure 1) is indistinguishable from the trajectory with $E^{\prime} / E=10^{-3}$.

\section{Numerical evaluation}

Let us now compare the analytic approximation above to a numerical solution of the full instanton equations (3.4). To fulfill the periodicity constraints, a shooting method can be used: we numerically integrate (3.4), varying the initial conditions and $a$ until a closed solution is found using a root finding algorithm. Figure 2 shows these closed solutions for different values of $\gamma_{\omega}$ and $E^{\prime} / E$ with a fixed $\gamma_{k}=0.5$. As explained above, for small $E^{\prime}$ the time dependent pulse acts like a reflecting barrier. For finite values of $E^{\prime}$ such as $E^{\prime} / E=10^{-1}$, this barrier is "softened" and the trajectories (blue curves in figure 2) differ a bit from the analytical approximation in figure 1 . For smaller $E^{\prime} / E$ however, they converge to the analytical approximation (orange and green curves in figure 2) and already for $E^{\prime} / E=10^{-3}$ (green curves in figure 2) they are virtually indistinguishable from the analytical approximation in figure 1 . They perfectly agree with the reflection picture, further justifying the approximations used above.

With these solutions to the instanton equations, we can evaluate the action (2.3). An exemplary section with $\gamma_{k}=0.3$ is shown in figure 3 , left panel. While the results for $E^{\prime} / E=10^{-1}$ (blue crosses) deviate a bit from the analytical approximation (red line) mainly because the total effective field is a bit larger than $E$, the agreement for $E^{\prime} / E=10^{-3}$ (green crosses) is excellent. Assembling all these sections for different values of $\gamma_{k}$ together, we obtain the landscape plot in figure 3 , right panel. Here we plotted the analytical result, but the landscape obtained numerically for $E^{\prime} / E=10^{-3}$, for example, would be indistinguishable. The red curve in figure 3, right panel, marks the threshold $\gamma_{\omega}^{\text {crit }}\left(\gamma_{k}\right)$ in (4.6). 

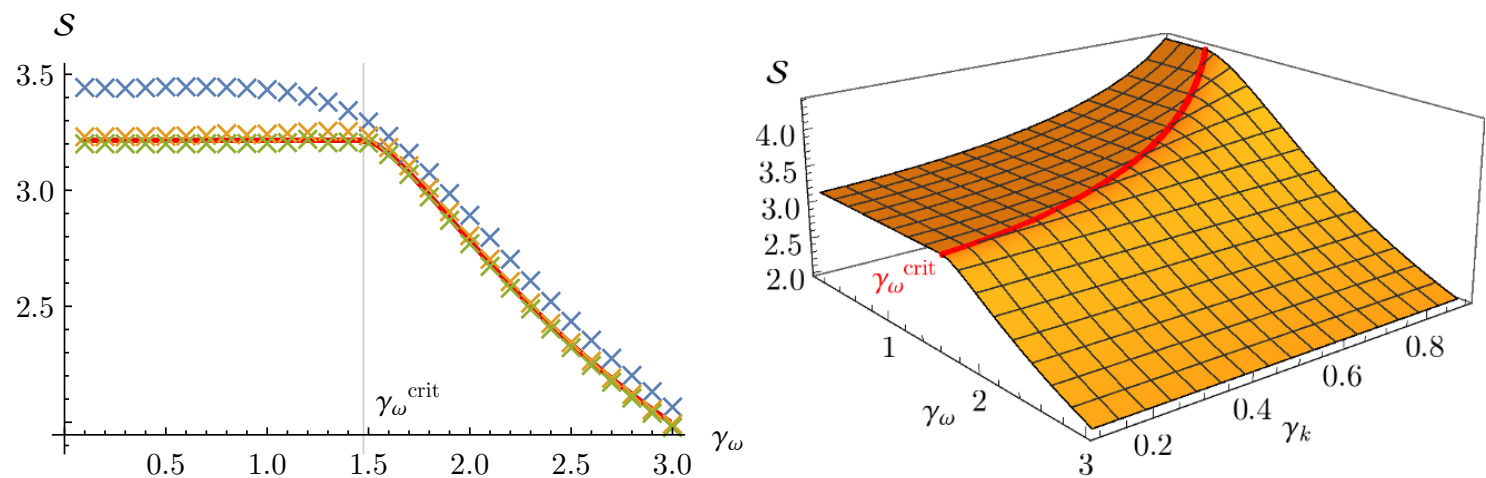

Figure 3. Left: comparison of the action (2.3) found by numerically integrating (3.4) for $\gamma_{k}=0.3$ and $E^{\prime} / E=10^{-1}$ (blue), $E^{\prime} / E=10^{-2}$ (orange), and $E^{\prime} / E=10^{-3}$ (green) to the integral (4.2) (red line). Right: (4.2) evaluated for varying $\gamma_{\omega}$ and $\gamma_{k}$. The thick, red line visualizes the threshold $\gamma_{\omega}^{\text {crit }}\left(\gamma_{k}\right)$ in (4.6). The action $\mathcal{S}$ is displayed in units of $m^{2} /(q E)$.

\section{Conclusions}

Via the world-line instanton technique, we derived an analytical estimate (4.2) for the electron-positron pair creation probability (2.2) induced by an electric field (3.1) which genuinely depends both on space and on time. Superimposing a strong spatial pulse by a weak temporal pulse (3.1), we found that the weak pulse is negligible for small frequencies $\gamma_{\omega} \leq \gamma_{\omega}^{\text {crit }}$ but can enhance the pair creation probability significantly (dynamically assisted Sauter-Schwinger effect) for larger frequencies $\gamma_{\omega}>\gamma_{\omega}^{\text {crit }}$ with the threshold (4.6) depending on the spatial Keldysh parameter (3.2). Note that we treated both, the strong $\propto E$ and the weak $\propto E^{\prime}$ field non-perturbatively. This becomes evident by the reflection of the instanton trajectory in figure 1 , for example, which is a drastic change and thus cannot be observed by a perturbative expansion around the strong field solution (see, e.g., [68]). Similarly, the space and time dependence is fully taken into account - the threshold behavior (4.6) requires going beyond the locally constant field approximation or gradient expansion (see, e.g., [72]).

In the homogeneous limit $\gamma_{k} \downarrow 0$, this threshold $\gamma_{\omega}^{\text {crit }}$ converges to $\pi / 2$ in accordance with [67]. If the spatial Keldysh parameter approaches unity $\gamma_{k} \uparrow 1$, on the other hand, the threshold $\gamma_{\omega}^{\text {crit }}$ goes to zero. In this case $\gamma_{k} \uparrow 1$, the size of the spatial Sauter pulse is barely enough to produce electron-positron pairs and the instanton loop becomes very large, cf. $x_{1}^{*}=\operatorname{artanh}\left(\gamma_{k}\right) / k$ for $b=0$. This equation $x_{1}^{*}=\operatorname{artanh}\left(\gamma_{k}\right) / k$ can be rewritten as $m=q A_{0}\left(x_{1}^{*}\right)$ which shows that the potential difference between the spatial turning points $+x_{1}^{*}$ and $-x_{1}^{*}$ equals the energy gap of $2 m c^{2}$. For $\gamma_{k} \uparrow 1$, the total asymptotic electrostatic potential difference approaches this energy gap and thus the spatial turning points $\pm x_{1}^{*}$ move to infinity. Quite intuitively, even comparably small frequencies (leading to poles at large distances to the origin) can have an impact in this limit.

Note that we focused on the exponent $\mathcal{S}$ in the electron-positron pair creation probability (2.2) given by the instanton action (2.3) in this work. The pre-factor in front of the exponential can also be derived (at least numerically) via the world-line instanton method 
by studying small perturbations around the instanton trajectory. ${ }^{3}$ Hence, this pre-factor is almost unaffected by the weak pulse $\propto E^{\prime}$ below threshold $\gamma_{\omega}<\gamma_{\omega}^{\text {crit }}$, but it is expected to depend on $E^{\prime}$ above threshold $\gamma_{\omega}>\gamma_{\omega}^{\text {crit }}$. Thus, for extremely small $E^{\prime}$, this pre-factor could partly counteract the exponential enhancement of the pair creation probability. However, since the parameter dependence (e.g., power-law) of this pre-factor is sub-dominant compared to the exponential dependence on $\mathcal{S}$, there will be a large region of parameter space where the dynamically assisted Sauter-Schwinger effect applies. ${ }^{4}$

In order to get a feeling for the orders of magnitude of the involved parameters, let us insert some potentially realistic example values. An optical laser focus with a focal field strength of $E=10^{16} \mathrm{~V} / \mathrm{m}$ corresponding to an intensity of order $10^{25} \mathrm{~W} / \mathrm{cm}^{2}$ has a very small $\gamma_{k}$. Hence we get $\gamma_{\omega}^{\text {crit }} \approx \pi / 2$ corresponding to a threshold frequency of $\omega^{\text {crit }} \approx 6 \mathrm{keV}$, which is well below the energy gap $2 m c^{2} \approx 1 \mathrm{MeV}$ and could be reached by an x-ray free-electron laser (XFEL) or by higher harmonic focusing [73], for example. If the strong field itself is generated by such high-energy sources, on the other hand, the resulting $\gamma_{k}$ can approach unity which lowers $\gamma_{\omega}^{\text {crit }}$ and thus $\omega^{\text {crit }}$. An XFEL with $12 \mathrm{keV}$, for example, has $\gamma_{k} \approx 1 / 2$ and thus we get $\omega^{\text {crit }} \approx 5 \mathrm{keV}$ while an XFEL with $24 \mathrm{keV}$ corresponds to $\gamma_{k} \approx 1$ such that $\omega^{\text {crit }}$ vanishes. In these cases, the actual frequency $\omega$ exceeds the threshold frequency $\omega^{\text {crit }}$ indicating that the dynamically assisted Sauter-Schwinger effect plays a role.

However, these numbers should only be understood as an illustration because the profile (3.1) does not portray a real laser pulse. This motivates the study of more realistic field configurations, for example transverse fields such as $A_{z}(t, x)$. Preliminary investigations indicate that the world-line instanton method can also be applied to these more complex field profiles and that the general behavior of dynamically assisted Sauter-Schwinger effect persists. This will be the subject of future studies, see also [74].

\section{Outlook}

Going a step beyond the scenario considered here, we may ask the question of what we can learn from this analysis for more general tunneling phenomena in the presence of additional time-dependent fields. One lesson might be that the characteristic time scale of the additional temporal dependence required for enhancing the tunneling probability is not set by the height of the tunneling barrier, for example, but by the imaginary turning time $x_{0}^{*}$ of the associated instanton solution (in our case $\omega x_{0}^{*}= \pm \pi / 2$ ). In non-relativistic quantum mechanics, this imaginary turning time has been identified with the traversal time for tunneling [75], i.e., the time the particle needs for tunneling through the barrier, but this identification is not undisputed.

Another lesson might be the relation between the final kinetic energy of the particle (after tunneling) and the turning points in space and (imaginary) time of the associated

\footnotetext{
${ }^{3}$ Note that, in contrast to the instanton action $\mathcal{S}$, the pre-factor does depend on the spin, i.e., may differ for scalar and spinor QED.

${ }^{4}$ If $E^{\prime}$ becomes too small, the approach used here breaks down eventually. On the one hand, the small value of $E^{\prime}$ invalidates the large- $m$ expansion (i.e., the instanton method), and, on the other hand, approximating $E^{\prime}$ by a classical field is no longer justified.
} 

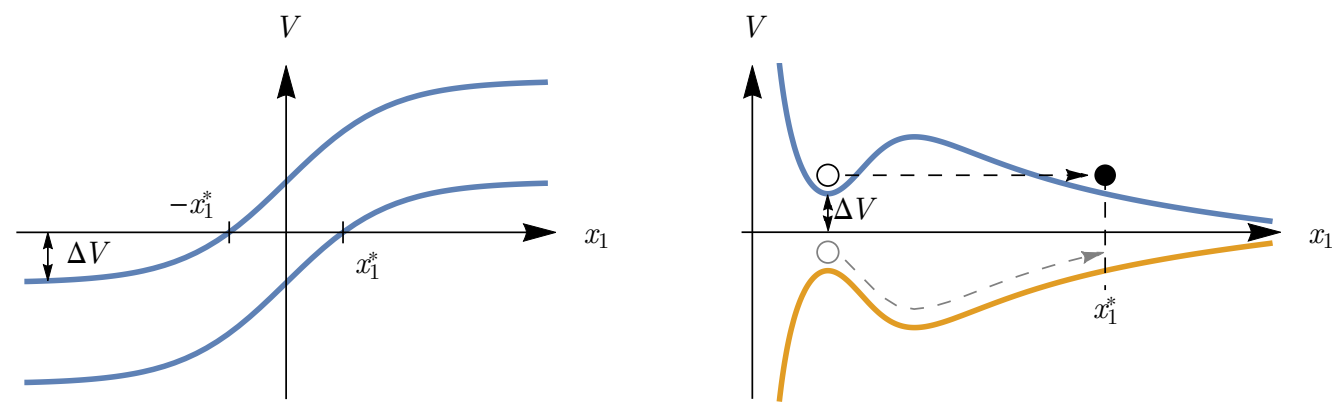

Figure 4. Sketch of the potential landscape $V(x)$ for the spatial Sauter profile $E(x)=E / \cosh ^{2}(k x)$ (left) in comparison to non-relativistic tunneling out of a local minimum (right), as well as the inverted potential $-V(x)$ which is "felt" by the non-relativistic instanton. In both cases, the spatial turning points $x_{1}^{*}$ move to infinity if the energy gain $\Delta V$ tends to zero.

instanton solution. For example, let us consider a general smooth and stationary potential landscape $V(x)$ which contains a local minimum and vanishes at spatial infinity, see figure 4. In non-relativistic quantum mechanics, the probability for tunneling from the local minimum out to infinity can also be estimated via an instanton solution, which basically corresponds to the motion of the particle within the inverted potential landscape $-V(x)$. Now, if the kinetic energy at infinity (i.e., $\Delta V$ ) becomes very small - which corresponds to the limit $\gamma_{k} \uparrow 1$ in the Sauter-Schwinger scenario - the spatial turning point $x_{1}^{*}$ and thus also the imaginary turning time $x_{0}^{*}$ become very large, suggesting that small $\omega$ can have a large impact.

As a potential application, one could envisage nuclear $\alpha$-decay, for example. In the usual Gamow picture [76], this process can be described by tunneling through a potential barrier which is dominated by the Coulomb repulsion far away from the nucleus while the nuclear attraction prevails at smaller distances. The height of this barrier is typically a few tens of $\mathrm{MeV}$ while its width, i.e., the spatial turning point $x_{1}^{*}$, depends on the final kinetic energy of the $\alpha$-particle $E_{\alpha}$.

Because the instanton trajectory grows very large for small $E_{\alpha} / m_{\alpha}$, the asymptotic behavior of the field (for large $x_{1}$ ) dominates since the "instanton spends most of its time there", see also [77]. We can calculate both the turning points and the instanton action to leading order in $E_{\alpha} / m_{\alpha}$ by considering an electric field that asymptotically corresponds to a Coulomb potential. Identifying $\gamma_{k}=\left(1+E_{\alpha} / m_{\alpha}\right)^{-1}$ the spatial turning point is

$$
x_{1}^{*}=\frac{q_{\alpha} q_{\text {nucleus }}}{4 \pi \varepsilon_{0} m_{\alpha}} \frac{1}{\gamma_{k}\left(1-\gamma_{k}\right)} \approx \frac{q_{\alpha} q_{\text {nucleus }}}{4 \pi \varepsilon_{0} E_{\alpha}},
$$

which is quite large (in comparison to other nuclear length scales) for typical decays. For example, ${ }^{238} \mathrm{U}$ has $E_{\alpha} \approx 4.3 \mathrm{MeV}$ and $x_{1}^{*} \approx 62 \mathrm{fm}$. The imaginary turning time is given by an integral similar to (4.5):

$$
x_{4}^{*} \approx \frac{m_{\alpha}}{q_{\alpha} E} \int_{0}^{1} \frac{\mathrm{d} u}{\left(1-\gamma_{k} \sqrt{1-u^{2}}\right)^{2}} \approx \frac{m_{\alpha}}{q_{\alpha} E} \frac{\pi}{\left(2 E_{\alpha} / m_{\alpha}\right)^{3 / 2}},
$$


so the critical threshold for dynamical assistance in this case is

$$
\omega^{\mathrm{crit}}=\frac{\pi}{2 x_{4}^{*}} \approx \frac{\pi}{Z} \frac{\varepsilon_{0} m_{\alpha}}{q^{2}}\left(2 \frac{E_{\alpha}}{m_{\alpha}}\right)^{3 / 2} .
$$

Again, for a typical decay, this is on the order of $100 \mathrm{keV}$ (e.g., for ${ }^{238} \mathrm{U}$, we get $\left.\omega^{\text {crit }}=157 \mathrm{keV}\right)$, far less then the other characteristic energy scales in the problem, such as the $\alpha$-particle mass, its final kinetic energy or the barrier height $Z q^{2} / 2 \pi \varepsilon_{0} R_{\text {nucleus }}$ $\left(\approx 35 \mathrm{MeV}\right.$ for $\left.{ }^{238} \mathrm{U}\right)$ used in the usual tunneling model.

Let us also calculate the instanton action (and thus the lifetime of the decay) analogously to (4.2). We have to slightly adapt the integral expression for the action in this case, because in the Sauter-Schwinger scenario (figure 4, left) there are two spatial turning points $\pm x_{1}^{*}$ moving to $\pm \infty$ as $\Delta V \rightarrow 0$, whereas for the $\alpha$-particle (figure 4 , right) there is only one $x_{1}^{*} \rightarrow \infty$. This amounts to a factor of $\frac{1}{2}$ in the leading-order expression for the instanton action:

$$
\begin{aligned}
\mathcal{S} & \approx \frac{1}{2} \frac{q_{\alpha} q_{\text {nucleus }}}{4 \pi \varepsilon_{0}} \frac{4}{\gamma_{k}^{2}} \int_{0}^{\frac{1}{1-\gamma_{k}}} \mathrm{~d} u \sqrt{\gamma_{k}^{2}-\left(1-\frac{1}{u}\right)^{2}} \\
& \approx \frac{q_{\alpha} q_{\text {nucleus }}}{2 \varepsilon_{0}} \frac{1}{\sqrt{2 E_{\alpha} / m_{\alpha}}} \\
& =\frac{q^{2} \sqrt{m_{\alpha}}}{\varepsilon_{0}} \frac{Z}{\sqrt{E_{\alpha}}}
\end{aligned}
$$

To leading order in $E_{\alpha} / m_{\alpha}$, we reproduce the well known Geiger-Nuttall law [78]

$$
\ln (P) \propto-\mathcal{S} \propto-\frac{Z}{\sqrt{E_{\alpha}}} .
$$

The expression (7.4) is also obtained as the leading-order contribution in the WKB formula for an $\alpha$-particle tunneling through a static Coulomb potential. Via the imaginary turning time in (7.2), we may now estimate the possibility of dynamically assisting this tunneling process.

Note that the mechanism sketched above is quite different from enhanced $\beta$-decay and similar ideas (see [79-87] for an incomplete selection) where the available phase space for the emitted or converted electrons or positrons is modified by the electromagnetic field.

Open Access. This article is distributed under the terms of the Creative Commons Attribution License (CC-BY 4.0), which permits any use, distribution and reproduction in any medium, provided the original author(s) and source are credited.

\section{References}

[1] F. Sauter, Über das Verhalten eines Elektrons im homogenen elektrischen Feld nach der relativistischen Theorie Diracs, Z. Phys. 69 (1931) 742 [INSPIRE].

[2] F. Sauter, Zum "Kleinschen Paradoxon", Z. Phys. 73 (1931) 547. 
[3] W. Heisenberg and H. Euler, Consequences of Dirac's theory of positrons, Z. Phys. 98 (1936) 714 [physics/0605038] [INSPIRE].

[4] V. Weisskopf, The electrodynamics of the vacuum based on the quantum theory of the electron, Kong. Dans. Vid. Selsk., Math.-fys. Medd. XIV (1936) 6, reprinted in: Quantum Electrodynamics, J. Schwinger eds., Dover, New York U.S.A. (1958).

[5] J.S. Schwinger, On gauge invariance and vacuum polarization, Phys. Rev. 82 (1951) 664 [INSPIRE].

[6] E. Brézin and C. Itzykson, Pair production in vacuum by an alternating field, Phys. Rev. D 2 (1970) 1191 [INSPIRE].

[7] N.B. Narozhnyi and A.I. Nikishov, The Simplist processes in the pair creating electric field, Yad. Fiz. 11 (1970) 1072 [Sov. J. Nucl. Phys. 11 (1970) 596] [InSPIRE].

[8] V.S. Popov, Production of e+e- Pairs in an Alternating External Field, JETP Lett. 13 (1971) 185 [hep-th/0107170].

[9] P. Kennedy, The Woods-Saxon potential in the Dirac equation, J. Phys. A 35 (2002) 689.

[10] D.D. Dietrich, Fermion production in time dependent fields, Phys. Rev. D 68 (2003) 105005 [hep-th/0302229] [INSPIRE].

[11] H. Gies and K. Klingmüller, Pair production in inhomogeneous fields, Phys. Rev. D 72 (2005) 065001 [hep-ph/0505099] [INSPIRE].

[12] S.P. Kim and D.N. Page, Improved Approximations for Fermion Pair Production in Inhomogeneous Electric Fields, Phys. Rev. D 75 (2007) 045013 [hep-th/0701047] [InSPIRE].

[13] H. Kleinert, R. Ruffini and S.-S. Xue, Electron-Positron Pair Production in Space- or Time-Dependent Electric Fields, Phys. Rev. D 78 (2008) 025011 [arXiv:0807.0909] [INSPIRE].

[14] F. Hebenstreit, R. Alkofer, G.V. Dunne and H. Gies, Momentum signatures for Schwinger pair production in short laser pulses with sub-cycle structure, Phys. Rev. Lett. 102 (2009) 150404 [arXiv: 0901.2631 ] [INSPIRE].

[15] C.K. Dumlu and G.V. Dunne, The Stokes Phenomenon and Schwinger Vacuum Pair Production in Time-Dependent Laser Pulses, Phys. Rev. Lett. 104 (2010) 250402 [arXiv: 1004.2509] [INSPIRE].

[16] C.K. Dumlu, Schwinger Vacuum Pair Production in Chirped Laser Pulses, Phys. Rev. D 82 (2010) 045007 [arXiv: 1006.3882] [INSPIRE].

[17] S.P. Kim and C. Schubert, Non-adiabatic Quantum Vlasov Equation for Schwinger Pair Production, Phys. Rev. D 84 (2011) 125028 [arXiv:1110.0900] [INSPIRE].

[18] C.K. Dumlu and G.V. Dunne, Interference Effects in Schwinger Vacuum Pair Production for Time-Dependent Laser Pulses, Phys. Rev. D 83 (2011) 065028 [arXiv:1102.2899] [INSPIRE].

[19] E. Akkermans and G.V. Dunne, Ramsey Fringes and Time-domain Multiple-Slit Interference from Vacuum, Phys. Rev. Lett. 108 (2012) 030401 [arXiv:1109.3489] [INSPIRE].

[20] E. Strobel and S.-S. Xue, Semiclassical pair production rate for time-dependent electrical fields with more than one component: WKB-approach and world-line instantons, Nucl. Phys. B 886 (2014) 1153 [arXiv:1312.3261] [INSPIRE].

[21] A.I. Nikishov and V.I. Ritus, Quantum Processes in the Field of a Plane Electromagnetic Wave and in a Constant Field 1, Sov. Phys. JETP 19 (1964) 529 [inSPIRE]. 
[22] A.I. Nikishov and V.I. Ritus, Pair production by a photon and photon emission by an electron in the field of an intense electromagnetic wave and in a constant field, Sov. Phys. JETP 25 (1967) 1135.

[23] T.N. Tomaras, N.C. Tsamis and R.P. Woodard, Back reaction in light cone QED, Phys. Rev. D 62 (2000) 125005 [hep-ph/0007166] [INSPIRE].

[24] T.N. Tomaras, N.C. Tsamis and R.P. Woodard, Pair creation and axial anomaly in light cone QED(2), JHEP 11 (2001) 008 [hep-th/0108090] [INSPIRE].

[25] H.M. Fried and R.P. Woodard, The One loop effective action of QED for a general class of electric fields, Phys. Lett. B 524 (2002) 233 [hep-th/0110180] [INSPIRE].

[26] F. Hebenstreit, A. Ilderton and M. Marklund, Pair production: the view from the lightfront, Phys. Rev. D 84 (2011) 125022 [arXiv:1109.3712] [INSPIRE].

[27] V. Dinu, T. Heinzl, A. Ilderton, M. Marklund and G. Torgrimsson, Vacuum refractive indices and helicity flip in strong-field QED, Phys. Rev. D 89 (2014) 125003 [arXiv:1312.6419] [INSPIRE].

[28] A. Ilderton, Localisation in worldline pair production and lightfront zero-modes, JHEP 09 (2014) 166 [arXiv:1406.1513] [INSPIRE].

[29] A. Ilderton, G. Torgrimsson and J. Wårdh, Nonperturbative pair production in interpolating fields, Phys. Rev. D 92 (2015) 065001 [arXiv:1506.09186] [INSPIRE].

[30] V.S. Popov, Pair Production in a Variable External Field (Quasiclassical approximation), Sov. Phys. JETP 34 (1972) 709.

[31] V.S. Popov and M.S. Marinov, e+e- pair production in variable electric field, Yad. Fiz. 16 (1972) 809 [INSPIRE].

[32] V.S. Popov, Resonant pair production in a strong electric field, JETP Lett. 18 (1973) 255.

[33] V.S. Popov, The imaginary time method for periodic fields, Sov. J. Nucl. Phys. 19 (1974) 584 .

[34] Y. Kluger, E. Mottola and J.M. Eisenberg, The Quantum Vlasov equation and its Markov limit, Phys. Rev. D 58 (1998) 125015 [hep-ph/9803372] [INSPIRE].

[35] F. Hebenstreit, R. Alkofer and H. Gies, Schwinger pair production in space and time-dependent electric fields: Relating the Wigner formalism to quantum kinetic theory, Phys. Rev. D 82 (2010) 105026 [arXiv: 1007.1099] [inSPIRE].

[36] F. Hebenstreit, R. Alkofer and H. Gies, Particle self-bunching in the Schwinger effect in spacetime-dependent electric fields, Phys. Rev. Lett. 107 (2011) 180403 [arXiv:1106.6175] [INSPIRE].

[37] F. Hebenstreit, A. Ilderton, M. Marklund and J. Zamanian, Strong field effects in laser pulses: the Wigner formalism, Phys. Rev. D 83 (2011) 065007 [arXiv:1011.1923] [INSPIRE].

[38] M. Ruf, G.R. Mocken, C. Müller, K.Z. Hatsagortsyan and C.H. Keitel, Pair production in laser fields oscillating in space and time, Phys. Rev. Lett. 102 (2009) 080402 [arXiv:0810.4047] [INSPIRE].

[39] M. Jiang et al., Electron-positron pair creation induced by quantum-mechanical tunneling, Phys. Rev. A 83 (2011) 053402.

[40] M. Jiang et al., Pair creation enhancement due to combined external fields, Phys. Rev. A 85 (2012) 033408. 
[41] F.V. Bunkin and I.I. Tugov, Possibility of creating electron-positron pairs in a vacuum by the focusing of laser radiation, Sov. Phys. Dokl. 14 (1970) 678.

[42] V. Ritus, Quantum effects of the interaction of elementary particles with an intense electromagnetic field, J. Sov. Laser Res. 6 (1985) 497.

[43] A. Nikishov, Problems of intense external-field intensity in quantum electrodynamics, J. Sov. Laser Res. 6 (1985) 619.

[44] A.D. Piazza, Pair production at the focus of two equal and oppositely directed laser beams: The effect of the pulse shape, Phys. Rev. D 70 (2004) 053013 [INSPIRE].

[45] S.S. Bulanov, N.B. Narozhny, V.D. Mur and V.S. Popov, On $e^{+} e^{-}$pair production by a focused laser pulse in vacuum, Phys. Lett. A 330 (2004) 1 [hep-ph/0403163] [INSPIRE].

[46] N.B. Narozhny, S.S. Bulanov, V.D. Mur and V. S. Popov, On e+e- pair production by colliding electromagnetic pulses, JETP Lett. 80 (2004) 382.

[47] S. Bulanov, N. Narozhny, V. Mur and V. Popov, Electron-positron pair production by electromagnetic pulses, JETP 102 (2006) 9.

[48] G.A. Mourou, T. Tajima and S.V. Bulanov, Optics in the relativistic regime, Rev. Mod. Phys. 78 (2006) 309 [INSPIRE].

[49] G.V. Dunne, New Strong-Field QED Effects at ELI: Nonperturbative Vacuum Pair Production, Eur. Phys. J. D 55 (2009) 327 [arXiv:0812.3163] [InSPIRE].

[50] S.S. Bulanov, T.Z. Esirkepov, A.G.R. Thomas, J.K. Koga and S.V. Bulanov, On the Schwinger limit attainability with extreme power lasers, Phys. Rev. Lett. 105 (2010) 220407 [arXiv: 1007.4306] [INSPIRE].

[51] L. Labun and J. Rafelski, Spectra of Particles from Laser-Induced Vacuum Decay, Phys. Rev. D 84 (2011) 033003 [arXiv: 1102.5773] [INSPIRE].

[52] R.P. Feynman, Mathematical formulation of the quantum theory of electromagnetic interaction, Phys. Rev. 80 (1950) 440 [InSPIRE].

[53] I.K. Affleck, O. Alvarez and N.S. Manton, Pair Production at Strong Coupling in Weak External Fields, Nucl. Phys. B 197 (1982) 509 [INSPIRE].

[54] S.P. Kim and D.N. Page, Schwinger pair production via instantons in a strong electric field, Phys. Rev. D 65 (2002) 105002 [hep-th/0005078] [INSPIRE].

[55] G.V. Dunne and C. Schubert, Worldline instantons and pair production in inhomogeneous fields, Phys. Rev. D 72 (2005) 105004 [hep-th/0507174] [INSPIRE].

[56] G.V. Dunne, Q.-h. Wang, H. Gies and C. Schubert, Worldline instantons. II. The Fluctuation prefactor, Phys. Rev. D 73 (2006) 065028 [hep-th/0602176] [INSPIRE].

[57] S.P. Kim and D.N. Page, Schwinger pair production in electric and magnetic fields, Phys. Rev. D 73 (2006) 065020 [hep-th/0301132] [INSPIRE].

[58] G.V. Dunne, Worldline instantons, vacuum pair production and Gutzwiller's trace formula, J. Phys. A 41 (2008) 164041 [inSPIRE].

[59] C.K. Dumlu and G.V. Dunne, Complex Worldline Instantons and Quantum Interference in Vacuum Pair Production, Phys. Rev. D 84 (2011) 125023 [arXiv:1110.1657] [INSPIRE]. 
[60] C. Schubert, Lectures on the worldline formalism, given at the School on Spinning Particles in Quantum Field Theory: Worldline Formalism, Higher Spins and Conformal Geometry, Morelia Mexico (2012), http://indico.cern.ch/event/206621/.

[61] L.V. Keldysh, Ionization in the field of a strong electromagnetic wave, Sov. Phys. JETP 20 (1965) 1307.

[62] D.L. Burke et al., Positron production in multi - photon light by light scattering, Phys. Rev. Lett. 79 (1997) 1626 [INSPIRE].

[63] W. Dittrich and H. Gies, Probing the quantum vacuum. Perturbative effective action approach in quantum electrodynamics and its application, Springer Tracts Mod. Phys. 166 (2000) 1.

[64] J. Avan, H.M. Fried and Y. Gabellini, Nontrivial generalizations of the Schwinger pair production result, Phys. Rev. D 67 (2003) 016003 [hep-th/0208053] [INSPIRE].

[65] G.V. Dunne, The search for the Schwinger effect: Nonperturbative vacuum pair production, Int. J. Mod. Phys. A 25 (2010) 2373 [InSPIRE].

[66] G.V. Dunne, The Heisenberg-Euler Effective Action: 75 years on, Int. J. Mod. Phys. A 27 (2012) 1260004 [arXiv:1202.1557] [INSPIRE].

[67] R. Schützhold, H. Gies and G. Dunne, Dynamically assisted Schwinger mechanism, Phys. Rev. Lett. 101 (2008) 130404 [arXiv:0807.0754] [inSPIRE].

[68] G.V. Dunne, H. Gies and R. Schützhold, Catalysis of Schwinger Vacuum Pair Production, Phys. Rev. D 80 (2009) 111301 [arXiv:0908.0948] [InSPIRE].

[69] A. Monin and M.B. Voloshin, Photon-stimulated production of electron-positron pairs in electric field, Phys. Rev. D 81 (2010) 025001 [arXiv:0910.4762] [InSPIRE].

[70] M. Orthaber, F. Hebenstreit and R. Alkofer, Momentum Spectra for Dynamically Assisted Schwinger Pair Production, Phys. Lett. B 698 (2011) 80 [arXiv:1102.2182] [INSPIRE].

[71] C. Fey and R. Schützhold, Momentum dependence in the dynamically assisted Sauter-Schwinger effect, Phys. Rev. D 85 (2012) 025004 [arXiv:1110.5499] [INSPIRE].

[72] H. Kleinert and S.-S. Xue, Electron-positron pair productions in classical electric field and electromagnetic wave, Annals Phys. 333 (2013) 104 [arXiv:1207.0401] [INSPIRE].

[73] S. Gordienko, A. Pukhov, O. Shorokhov and T. Baeva, Coherent Focusing of High Harmonics: A New Way Towards the Extreme Intensities, Phys. Rev. Lett. 94 (2005) 103903 [INSPIRE].

[74] M.F. Linder, C. Schneider, J. Sicking, N. Szpak and R. Schützhold, Pulse shape dependence in the dynamically assisted Sauter-Schwinger effect, Phys. Rev. D 92 (2015) 085009.

[75] M. Büttiker and R. Landauer, Traversal Time for Tunneling, Phys. Rev. Lett. 49 (1982) 1739 [INSPIRE].

[76] G. Gamow, Zur Quantentheorie des Atomkernes, Z. Phys. 51 (1928) 204.

[77] H. Gies and G. Torgrimsson, Critical Schwinger pair production, arXiv:1507.07802 [INSPIRE].

[78] H. Geiger and J.M. Nuttall, The ranges of the $\alpha$ particles from various radioactive substances and a relation between range and period of transformation, Philos. Mag. Ser. 622 (1911) 613 [Erratum ibid. 23 (1912) 439]. 
[79] W. Becker, W.H. Louisell, J.D. McCullen and M.O. Scully, Laser Enhancement of Nuclear $\beta$ Decay, Phys. Rev. Lett. 47 (1981) 1262.

[80] J.I. Gersten and M.H. Mittleman, Comment on "Laser Enhancement of Nuclear $\beta$ Decay", Phys. Rev. Lett. 48 (1982) 651.

[81] H.R. Reiss, Laser Enhancement of Nuclear $\beta$ Decay, Phys. Rev. Lett. 48 (1982) 652.

[82] W. Becker, W.H. Louisell, J.D. McCullen and M.O. Scully, Becker et al. Respond, Phys. Rev. Lett. 48 (1982) 653.

[83] G.C. Baldwin and S.A. Wender, Effect of Laser Radiation on the Decay of U, Phys. Rev. Lett. 48 (1982) 1461.

[84] H.R. Reiss, Nuclear beta decay induced by intense electromagnetic fields: Basic theory, Phys. Rev. C 27 (1983) 1199 [InSPIRE].

[85] W. Becker, R.R. Schlicher and M.O. Scully, Comment on enhancement of forbidden nuclear beta decay by high-intensity radio-frequency fields, Phys. Rev. C 29 (1984) 1124 [INSPIRE].

[86] H.R. Reiss, Reply to "Comment on enhancement of forbidden nuclear beta decay by high-intensity radio-frequency fields", Phys. Rev. C 29 (1984) 1132 [INSPIRE].

[87] J.L. Friar and H.R. Reiss, Modification of nuclear $\beta$ decay by intense low-frequency electromagnetic waves, Phys. Rev. C 36 (1987) 283 [INSPIRE]. 